\title{
Features of the direct flexoeffect in cholesterics and twist-structures of nematics
}

\author{
O. A. Denisova ${ }^{1, \dagger}$, O. A. Skaldin ${ }^{2}$ \\ †denisovaolga@bk.ru \\ ${ }^{1}$ Ufa State Petroleum Technological University, 1 Kosmonavtov str., 450062, Ufa, Russia \\ ${ }^{2}$ Institute of Molecule and Crystal Physics Ufa Research Center of RAS, 151 pr. Oktaybrya, 450075, Ufa, Russia
}

\begin{abstract}
Specific features of the direct flexoelectric effect in cholesteric liquid crystals (LC) MBBA + approx. $2 \%$ cholesteryl chloride and twist structures of nematic liquid crystals under periodic shear oscillations are studied experimentally. The cell represented an assembly of three plates the lower and upper ones of which were separated by spacers of a given thickness, whereas the central one floated freely in the LC. Two methods of oscillation excitation in an LC cell [3 - 5] were used. To observe the flexoeffect, excitation of a transverse shear wave was initiated by longitudinal oscillations of one of the glass substrates coating the LC layer. The registration of a polarization voltages $U_{1}$ и $U_{2}$ induced in the LC layer by a periodic deformation of the director field was carried out by two ways: record of the voltage $U_{2}$ between the movable plate and fixed substrate and registration of voltage $\mathrm{U}_{1}$ on the fixed substrate. The dependences of the director deflection angle and magnitude of the flexoelectric effect on the plate oscillation rate obey a linear relationship that is explained by the Eriksen-Leslie theory. The frequency dependences of the flexoelectric polarization induced by the periodic shift, which has a resonance character, are experimentally obtained. It is shown that the resonance character of the detected potential difference $U_{1}$ и $U_{2}$ is related to the scattering of the propagating elastic-viscous wave by the helicoidal twist structure formed in the nematic and weak cholesteric.
\end{abstract}

Keywords: cholesteric liquid crystals, twist-structure of nematics, flexoelectrical effect.

\section{Особенности проявления прямого флексоэффекта} в холестериках и твист-структурах нематиков

\author{
Денисова О.А. ${ }^{1, \dagger}$, Скалдин О. А. ${ }^{2}$
}

${ }^{1}$ Уфимский государственный нефтяной технический университет, ул. Космонавтов, 1, 450062, Уфа, Россия ${ }^{2}$ Институт физики молекул и кристаллов УНЦ РАН, пр. Октября 151, 450075, Уфа, Россия

Экспериментально изучены особенности прямого флексоэлектрического эффекта в холестерических жидких кристаллах (ЖК) МББА + ок. 2\% холестерилхлорида и твист-структурах нематических жидких кристаллов под действием периодических сдвиговых осцилляций. Ячейка представляла собой сборку их трех пластин. Нижняя и верхняя пластины были разделены прокладками заданной толщины, а центральная свободно плавала в ЖК. В работе использовались два метода возбуждения колебаний в ЖК-ячейке [3-5]. Для наблюдения флексоэффекта возбуждение поперечной волны осуществлялось продольными колебаниями одной из ограничивающих ЖК-слой стеклянных подложек. Сдвиговые колебания рабочей пластины возбуждались электродинамическим вибратором через волновод вдоль направления, коллинеарного плоскости подвижной пластины, что позволяло изучать отклик ЖК-ячейки на внешнее воздействие в широком диапазоне звуковых частот 20 - 20000 Гц. Регистрация разностей потенциалов $U_{1}$ и $U_{2}$ индуцируемых в слое ЖК за счет периодической деформации поля директора проводилась двумя способами: регистрацией напряжения: $U_{2}$ между подвижной и неподвижной пластинами с нанесенным на них проводником, и измерением напряжения $\mathrm{U}_{1}$ на проводнике неподвижной пластины. Зависимости угла отклонения директора и величины флексоэлектрического эффекта от скорости колебания пластины имеют линейный характер, что объясняется теорией Эриксена - Лесли. Экспериментально получены частотные зависимости флексоэлектрической поляризации, индуцируемой периодическим сдвигом, которые имеют резонансный характер. Показывается, что резонансный характер поведения регистрируемой разности потенциалов связан с рассеянием распространяющейся упруго-вязкой волны на геликоидальной твист-структуре нематика и слабого холестерика.

Ключевые слова: холестерические жидкие кристаллы, твист-структуры нематиков, флексоэлектрический эффект. 


\section{1. Введение}

При колебательном движении пластины в жидкости, ограниченной твердой поверхностью, возникает поперечная волна с глубиной проникновения $\delta$. Эта глубина падает с ростом частоты и растет с увеличением вязкости. В жидком кристалле (ЖК) при низкочастотных колебаниях возникают еще и колебания, связанные с изменением ориентации молекул [1]. Однако глубина проникания такой волны значительно меньше глубины проникания вязкой поперечной волны и составляет микроны. Особенности холестерических жидких кристаллов (ХЖК) и твист-структур состоят в том, что они подобны твердым телам из-за наличия спиральной структуры. Основную роль играет упругая деформация оси структуры, а не процессы, связанные с вязкостью [2]. Глубина проникания волны до частот $10^{6}$ Гц составляет порядка размеров толщины образца, но не смотря на это они могут инициировать ориентационные превращения и образование доменных структур [3]. В целом имеет место традиционный подход к изучению данных систем по схеме акустическое воздействие-оптический сигнал или изменение структуры. В данной же работе предлагается, ко всему прочему, и изучение электрического отклика.

Актуальность изучения вопроса распространения поперечных волн в жидких кристаллах связана с перспективами практического применения твист-ячеек в технике, например, для создания дисплеев нового поколения. Применение жидких кристаллов означает экономическую эффективность, простоту, удобство, небольшие габариты устройств и малые потребляемые мощности. Кроме того, например, управляемые оптические транспаранты могут быть использованы не только как элементы проекционного устройства, но и выполнять значительное число функций, связанных с преобразованием, хранением и обработкой оптических сигналов.

В связи с этим в настоящей работе исследованы ориентационные процессы, связанные с распространением поперечной вязкоупругой волны в твист-структурах нематических жидких кристаллов и холестерических жидких кристаллах с большим шагом спирали. Другой важной задачей было выяснения особенностей проявления флексоэлектрического эффекта, индуцированного поперечной волной в этих молекулярных системах, и влияние на него взаимодействия сдвиговой волны со спиральной структурой ЖК.

\section{2. Теория распространения упруго-вязкой волны в твист-структурах ЖК}

Распространение поперечной волны, возбуждаемой, подвижной пластиной в холестерическом жидком кристалле с осью спирали, параллельной оси $O Z$ и перпендикулярной вектору скорости $\vec{v}_{0}$, в среде будет описываться уравнениями Навье-Стокса для скоростей центров инерции молекул:

$$
\rho \frac{d v_{i}}{d t}=-\frac{\partial p}{\partial x_{i}}+\frac{\partial \sigma_{i j}^{\prime}}{\partial x_{j}}
$$

$v_{i}$ - скорости центров инерции молекул,

$$
\begin{gathered}
\sigma_{i j}^{\prime}=\alpha_{1} n_{k} n_{p} A_{k p} n_{i} n_{j}+\alpha_{2} n_{i} N_{j}+\alpha_{3} n_{j} N_{i}+ \\
+\alpha_{4} A_{i j}+\alpha_{5} n_{i} n_{k} A_{k j}+\alpha_{6} n_{j} n_{k} A_{k i}
\end{gathered}
$$

$\alpha_{1}, \ldots, \alpha_{6}-$ коэффициенты вязкости, $n_{k}-$ компоненты директора,

$$
A_{k j}=\frac{1}{2}\left(\frac{\partial v_{k}}{\partial x_{j}}+\frac{\partial v_{j}}{\partial x_{k}}\right), \quad \vec{N}=\frac{d \vec{n}}{d t}+[\vec{n} \times \operatorname{rot} \vec{v}],
$$

p - давление, моментные уравнения, описывающие движение директора:

$$
h_{\mu}=\gamma_{2} n_{\alpha} A_{\alpha \mu}+\gamma_{1} N_{\mu},
$$

где $h_{\mu}=-\partial F / \partial n_{\mu}-$ молекулярное поле, свободная энергия $F$ равная [1]:

$$
\begin{gathered}
F=-\frac{1}{2} \int\left\{K_{11}(\operatorname{div} \vec{n})^{2}+K_{22}\left(\vec{n} \operatorname{rot} \vec{n}+\vec{q}_{0}\right)^{2}+\right. \\
\left.+K_{33}[\vec{n} \times \operatorname{rot} \vec{n}]^{2}\right\} d v,
\end{gathered}
$$

здесь $K_{11}, K_{22}, K_{33}-$ модули упругости ЖК.

Выбирая возмущения директора $\delta n_{x}, \delta n_{y}, \delta n_{z}$ малыми, имеем:

$$
\begin{gathered}
n_{x} \approx n_{x}^{0}+\delta n_{x}, \quad n_{y} \approx n_{y}^{0}+\delta n_{y}, \quad n_{z} \approx n_{z}^{0}+\delta n_{z}, \\
n_{x}^{0}=\cos q_{0} z, \quad n_{y}^{0}=\sin q_{0} z, \quad n_{x}=\cos \theta, \quad n_{y}=\sin \theta, \\
n_{z}=\theta, \quad \theta=q_{0} t+C, \quad v_{x}=v_{0} \exp (i \omega t) .
\end{gathered}
$$

Будем рассматривать случай малых амплитуд колебаний и считать ориентацию директора «замороженной», т.е. $\delta n_{x}=\delta n_{y}=\delta n_{z} \approx 0$. В этом случае волна будет чисто вязкой, но с отличными от нуля компонентами скорости $v_{x}, v_{y}$, возбуждаемых одномерным сдвигом, например, оси OX. С учетом этого, из (1) и (2), исключая давление перекрестным дифференцированием, для уравнений движения центров инерции молекул и уравнение движения $z$-компоненты директора, описывающее колебание оси спирали, получаем:

$$
\begin{aligned}
\rho \frac{\partial v_{x}}{\partial t}= & \eta_{1} \frac{\partial}{\partial z}\left(\sin 2 q_{0} z \frac{\partial v_{y}}{\partial z}\right)+ \\
& +\eta_{2} \frac{\partial}{\partial z}\left(\cos 2 q_{0} z \frac{\partial v_{z}}{\partial z}\right)+\eta_{3} \frac{\partial^{2} v_{x}}{\partial z^{2}}, \\
\rho \frac{\partial v_{y}}{\partial t}= & \eta_{4} \frac{\partial}{\partial z}\left(\sin 2 q_{0} z \frac{\partial v_{x}}{\partial z}\right)+ \\
& +\eta_{2} \frac{\partial}{\partial z}\left(\cos 2 q_{0} z \frac{\partial v_{y}}{\partial z}\right)+\eta_{3} \frac{\partial^{2} v_{y}}{\partial z^{2}}, \\
\gamma_{2} n_{y}^{0} A_{y z} & +\gamma_{2} n_{x}^{0} A_{x z}+\gamma_{1} \frac{\partial\left(\delta n_{z}\right)}{\partial t} \approx 0 .
\end{aligned}
$$

В окрестности значений волнового вектора попереч- 
ной волны, сравнимых с шагом спирали $\mathrm{k} 2 \mathrm{qo}$, решение (4) запишется в виде:

$$
\begin{aligned}
& v_{x}=\left(C_{11}+C_{12} e^{i 2 q_{0} z}+C_{13} e^{i k^{\prime} z}+C_{14} e^{-i\left(k^{\prime}-2 q_{0}\right) z}\right) e^{-(\beta z-i \omega t)}, \\
& v_{y}=\left(C_{21}+C_{22} e^{i 2 q_{0} z}+C_{23} e^{i k^{\prime} z}+C_{24} e^{-i\left(k^{\prime}-2 q_{0}\right) z}\right) e^{-(\beta z-i \omega t)},
\end{aligned}
$$

где $k=k^{\prime}+i \beta, k^{\prime}-$ волновой вектор, $\beta-$ коэффициент затухания волны.

Нетривиальное решение системы уравнений будет при равенстве нулю детерминанта $\operatorname{det}\left|a_{l j}\right|=0, j=1, \ldots 4$, $a_{11}=a_{22}=a_{33}=a_{44}=\eta_{2} k^{\prime}\left(\mathrm{k}^{\prime}-2 q_{0}\right), a_{12}=a_{21}=a_{34}=a_{43}=\eta_{3} k^{\prime}+i \rho \omega$, $a_{13}=a_{24}=a_{31}=i \eta_{1} k^{\prime}\left(k^{\prime}-2 q_{0}\right), a_{14}=a_{23}=a_{32}=a_{41}=0$.

Откуда при $k^{\prime} \sim 2 q_{0}$, имеют место соотношения:

$$
\begin{gathered}
k^{\prime}=r^{1 / 2} \sin \varphi / 2, \\
\beta=\frac{\eta_{3} q_{0}}{\eta_{2}+\eta_{3}}-r^{1 / 2} \cos \varphi / 2, \\
r^{2}=\frac{16 \eta_{2}^{4} q_{0}^{4}}{\left(\eta_{2}+\eta_{3}\right)^{4}}+\frac{\rho^{2} \omega^{2}}{\left(\eta_{2}+\eta_{3}\right)^{2}}, \\
\operatorname{tg} \varphi=\frac{\rho \omega}{\eta_{2} q_{0}} .
\end{gathered}
$$

При постоянной скорости сдвига верхней пластины $v_{0}=$ const,$\beta=q_{0} \eta_{2} /\left(\eta_{2}+\eta_{3}\right)$, а $k^{\prime}=0$, что совпадает с известными результатами [1]. Так как на границах при $z=0$, $v=0$, а при $z=\sigma, v=v_{0} \exp (i \omega t)$, то значения скоростей с учетом (6) имеют вид:

$$
\begin{aligned}
& v_{x}=\left(C_{11}+C_{12} e^{i 2 q_{0} z}+C_{13} e^{i k^{\prime} z}+C_{14} e^{-i\left(k^{\prime}-2 q_{0}\right) z}\right) e^{-(\beta z-i \omega t)}, \\
& v_{y}=\left(C_{21}+C_{22} e^{i 2 q_{0} z}+C_{23} e^{i k^{\prime} z}+C_{24} e^{-i\left(k^{\prime}-2 q_{0}\right) z}\right) e^{-(\beta z-i \omega t)},
\end{aligned}
$$

Из выражения (7), в частности, следует, что возникающая волна является эллиптической, а в окрестности значений волновых векторов $k^{\prime} \sim 2 q_{0}$ имеет место резонансное увеличение скоростей, т.е. фактически имеет место резонанс оси спирали. Условия резонансов можно записать следующим образом:

$$
k^{\prime}-2 q_{0} \approx n \pi / \sigma, n=0,1,2, \ldots
$$

При выполнении этого соотношения происходит так же смена фазы регистрируемого сигнала относительно фазы подвижной пластины на $2 \pi$.

\section{3. Методика эксперимента}

Для экспериментальной оценки величин сигналов флексоэлектрического отклика в окрестности $q_{0}$ можно использовать связь градиентов скоростей и величины пьезоэлектрической поляризации $P_{i}$, определяемых пьезокоэффициентами $C_{i j k}$ по теории Проста [4]:

$$
P_{i}=C_{i j k} d v_{j} / d x_{k} .
$$

Необходимо отметить, что флексоэлектрический эффект является разновидностью пьезоэлектрического эффекта для анизотропных жидкостей, каковыми и являются жидкие кристаллы.

Симметрии ХЖК и твист-структур НЖК допускают только два отличных от нуля пьезоэлектрических коэф- фициента $C_{x y z}$ и $C_{y x z}$. Для величин поляризации, возникающих вдоль осей $O X$ и $O Y$, имеем:

$$
P_{x}=C_{x y z} d v_{y} / d z, \quad P_{y}=C_{y x z} d v_{x} / d z .
$$

Эти соотношения позволяют сделать оценки коэффициентов $C_{x y z}, C_{y x z}$ путем измерения $P_{x}$ и $P_{y}$ в зависимости, например, от величины волновых векторов ХЖК-спирали или частоты колебаний пластины. Методы измерения величины пьезоэлектрического отклика и распределение директора в объеме подробно описаны в работах [5-7]. Кроме исследования разности потенциалов, индуцируемой сдвигом, проводились измерения ее фазы относительно опорного сигнала, регистрируемого с датчика скорости движения пластины. Опорный сигнал поступал с датчика скорости, усиливался селективным усилителем и через пробник поступал на второй вход измерителя разности фаз. Сигнал, пропорциональный разности фаз, анализировался аналого-цифровым преобразователем и обрабатывался с помощью компьютера. Проводилось измерение полезного сигнала двумя методами: либо измерялось напряжение $U_{2}$ между подвижной пластиной и одним из электродов, либо снимался сигнал с пленочного сопротивления, наносимого на подвижную пластину $U_{1}$.

Были проведены измерения пьезоэлектрического отклика от толщины образцов, однако, какой-либо его зависимости обнаружено не было (до 5 мкм). Этот результат свидетельствует о быстром затухании сдвиговой волны в глубину кристалла, что вытекает из оценок эффективной глубины затухания сдвиговой волны в НЖК, проводимой по теории Эриксена-Лесли [5]. На рис. 1 приведена зависимость величины флексоэлектрического эффекта от скорости смещения пластины. Из этой зависимости вытекает, что при $v \approx 10^{-2} \mathrm{M} / \mathrm{c}$ индуцируемый сигнал линейно зависит от скорости.

При этом угол отклонения директора, определенный из фотоупругого эффекта, линейно зависит от скорости сдвига (рис. 2). Последнее находит объяснение в рамках теории Эриксена - Лесли [5].

Для исследования особенностей флексоэффекта был выбран жидкий кристалл $n$-метоксибензилиден-бутиланилин (МББА), в который сделана добавка оптически активного вещества, холестерилхлорида $\approx 2 \%$, так что величина волнового вектора ХЖК была $3 \times 10^{5} \mathrm{M}^{-1}$.

\section{4. Результаты и их обсуждение}

Рассмотрим величину флексоэлектрического сигнала и его изменение при постоянной скорости сдвига центральной пластины. Для получения различных волновых векторов $k^{\prime}$ поперечной вязкой волны при этом достаточно менять частоту сдвига. Поэтому рассмотрим более подробно частотную зависимость индуцированной сдвигом ЭДС на частоте первой гармоники, измеряемой на пленочном сопротивлении - электроде неподвижной верхней подложки. Полученная зависимость имеет типичный резонансный характер (рис. 3), определяется, по-видимому, спиральностью ЖК-струк- 
туры $[5,7]$. Эта ситуация возможна, когда длина волны, распространяющейся в НЖК, сравнима с шагом спирали. С уменьшением шага спирали резонансы зарегистрировать не удалось.

С целью дальнейшего уточнения соотношения (7) изучались твист-структуры НЖК, полученные в ячейках с планарной ориентацией молекул на поверхности. Для получения твист-структур опорные пластины, поворачивались относительно друг друга на $90^{\circ}$. Однако здесь основной проблемой стало получение планарных слоев холестерического жидкого кристалла. Для решения этого вопроса применялся метод растяжения слоя с одновременной закруткой пластин.

При полном повороте пластин на $360^{\circ}$ возникала ориентация молекул холестерика с шагом спирали, кратной толщине исследуемого кристалла, с планарной ориентацией молекул на границах.

Согласно соотношениям (7) при большом шаге спирали, т. е. когда $q_{0}^{2}<<\omega / \eta$, резонансы должны возникать при более высокой частоте. Результаты экспериментов полностью подтвердили этот факт: наличие закрученности в НЖК приводит к резонансным явлениям при распространении поперечных волн с длинами $\lambda$, сравнимыми с размерами образца ( $h \approx 10-15$ мкм), и получаемых при более высоких частотах колебаний. Явление резонанса весьма чувствительно к плоскопараллельности кюветы ячейки, в которую был помещен НЖК.

Как уже отмечалось, в нашем случае возможно изменение сдвига фаз $\Delta \varphi$ между сигналом, возбуждающим колебание, и регистрируемым сигналом $U_{1 \omega}$. Установлено, что при изменении частоты колебаний подвижной пластины изменяется и разность фаз $\Delta \varphi$. Такое изменение следует из (7), т. к. разность фаз $v_{x}-$ и $v_{y}$-компонент:

$$
\Delta \varphi \sim \frac{v_{x}}{v_{y}} \sim \operatorname{ctg}\left(k^{\prime}-2 q_{0}\right) \sigma .
$$

То есть в случае жидкокристаллических структур с пространственной дисперсией наблюдается резонансный характер изменения скорости $\Delta v$ от частоты, причем, вблизи резонансов имеет место скачок фазы на $2 \pi$ (рис. 4).

\section{5. Заключение}

Итак, при распространении поперечной вязкой волны в нематохолестерическом жидком кристалле с большим шагом спирали под действием периодического сдвига звукового диапазона индуцируется волна с малым волновым вектором $(k-2 q)$, что приводит к резонансному изменению скорости ориентационных волн при изменении частоты их генерации. Согласно теории Проста [4] флексополяризация в холестерических системах определяется градиентом скорости потока, что приводит к резонансному поведению ре- гистрируемого сигнала - переменного напряжения от индуцируемой флексодеформации. Последнее подтверждается экспериментальными данными при изучении, в том числе, и закрученных твист-слоев нематиков.

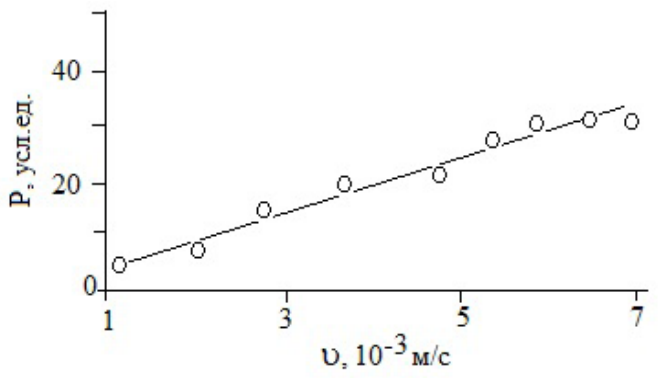

Рис. 1. Зависимость величины индуцированной флексоэлектрической поляризации от скорости колебания пластины.

Fig. 1. The dependence of the induced polarization flexoelectrical on the speed fluctuation of the plate.

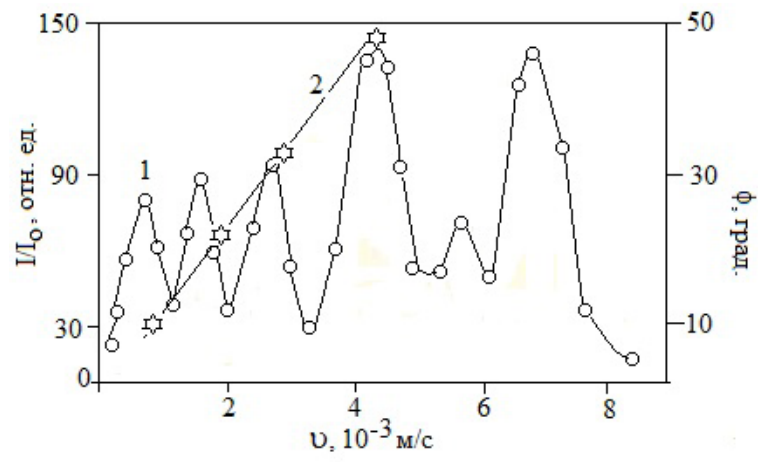

Рис. 2. Зависимости: 1 - интенсивности поляризованного света при фотоупругом эффекте; 2 - среднего угла отклонения директора от скорости колебания пластины.

Fig. 2. Dependencies: $1-$ the intensity of light at the photoelastic effect, 2 - director of the deflection angle of the speed fluctuations of the plate.

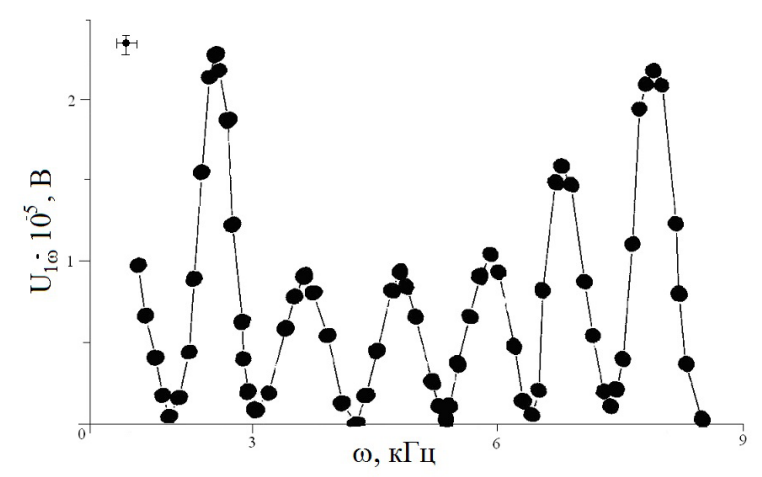

Рис. 3. Частотная зависимость первой гармоники $U_{1 \omega}$ в холестерическом жидком кристалле.

Fig. 3. Frequency dependence of the first $U_{1 \omega}$ harmonics in a cholesteric liquid crystal. 


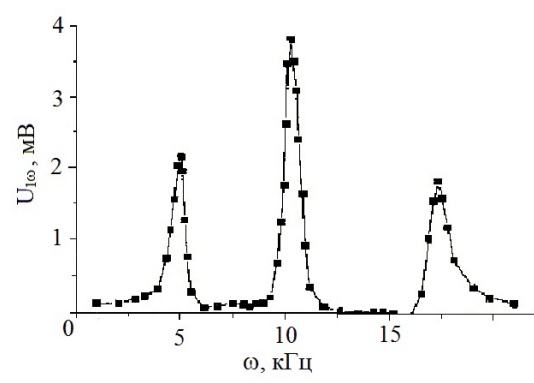

a

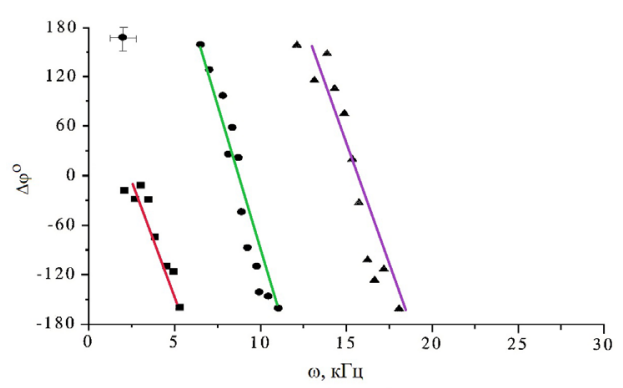

$\mathrm{b}$

Pис. 4. Частотные зависимости а) флексоэлектрического сигнала $U_{1 \omega}$; b) сдвига фаз $\Delta \varphi$ в закрученном нематике $\left(\right.$ МББА, $\left.t_{N}=25^{\circ} \mathrm{C}\right)$.

Fig. 4. Frequency dependence of a) flexoelectrical $U_{1 \omega}$ signal; b) phase shift $\Delta \varphi$ in a twisted nematic $\left(\mathrm{MBBA}, t_{N}=25^{\circ} \mathrm{C}\right)$.

\section{Литература/References}

1. А.П. Капустин, О.А. Капустина Акустика жидких кристаллов Наука. М. Гл. ред. физ.-мат. лит. (1986). 247 c.]

2. О.А. Капустина Кристаллография, 2014, том 59, № 4, c. $627-634$

3. О.А. Капустина Акустический журнал, 2015, том 61, № 1, c. $49-56$

4. J. Prost. Le Journal de Physique. 6 (39), 639 (1978).

5. O.A. Denisova, O.A. Scaldin. Letters on materials 6 (3),
168 (2016) (in Russian) [О. А. Денисова, О. А. Скалдин. Письма о материалах. 6 (3), 168 (2016).]

6. O.A. Denisova. Electrotechnical and information complexes and systems. 2 (9), 107 (2013) (in Russian) [О.А. Денисова. Электротехнические и информационные комплексы и системы. 2 (9), 107 (2013).]

7. O.A. Denisova, O.A. Scaldin. Electrotechnical and information complexes and systems. 4 (9), 145 (2013) (in Russian) [О.А. Денисова, О.А. Скалдин. Электротехнические и информационные комплексы и системы. 4 (9), 145 (2013).] 